Letter

\section{The role of Emergency Departments for Antimicrobial stewardship in COVID-19 Pandemic; the time is now}

\author{
Madiha Ismail ${ }^{1}$, Nazeer Najeeb Kapadia², \\ Sara Usman ${ }^{3}$
}

How to cite this:

doi: https://doi.org/10.12669/pjms.38.1.5107

Ismail M, Kapadia NN, Usman S. The role of Emergency Departments for Antimicrobial stewardship in COVID-19 Pandemic; the time is now. Pak J Med Sci. 2022;38(1):329. doi: https://doi.org/10.12669/pjms.38.1.5107

Inadvertent use of antibiotics is a primary driver of antimicrobial resistance globally and posing a significant risk to patient safety. With scarce treatment options and considerable overlap of symptoms between COVID-19 and bacterial pneumonia, the reflex prescription of antibiotics has become a routine. ${ }^{1}$ The situation is even more alarming in lower-middle-income countries where multi-resistant organisms are rampant and over the counter prescription of restricted antibiotics is considered a norm; knowledge gaps of healthcare providers and lack of governmental policies and surveillance are equally at fault here. ${ }^{2}$

The WHO guidelines for the clinical management of COVID-19 advise clinicians to start empirical antimicrobial treatment only in severe cases. Bacterial co-infections are quite rare and reported as low as $3.5 \%$ internationally. Recent systematic reviews identified that $72 \%$ of patients with COVID-19 receive antibiotic therapy even though only $7 \%$ having a bacterial co-infection. ${ }^{3}$ In a casecontrol study from Pakistan, Nasir $\mathrm{N}$ et al. reported an overall antibiotic utilization at $82 \% ; 64 \%$ in patients with no evidence of bacterial infection. ${ }^{4}$

1. Dr. Madiha Ismail, FCPS.

2. Dr. Nazeer Najeeb Kapadia, FCPS.

3. Dr. Sara Usman,

1-3: Department of Emergency Medicine,

Aga Khan University Hospital, Karachi, Pakistan.

Correspondence:

Dr. Madiha Ismail, FCPS.

Assistant Professor, Department of Emergency Medicine, Aga Khan University Hospital, Stadium Road, Karachi. Email: Madiha.ismail@aku.edu

* Received for Publication:

August 1, 2021

* Accepted for Publication:
October 25,2021
In the developed world, systemic antibiotics related adverse events account for an estimated 142,500 ED visits each year. Emergency Department(ED) is an interface between the inpatient and community settings. The antibiotic choice made by the ED physician also influences the therapy continued in the inpatient setting. Also, ED plays a vital role in obtaining relevant cultures before administering antibiotics to tailor or stop antibiotic therapy during hospitalization. Globally, ED practitioners must acknowledge their role to address the increasing problem of antimicrobial resistance. ${ }^{5}$

Even if emergency care providers appreciate the public health implications of growing antimicrobial resistance, changing their practices in the ED is challenging due to frequent interruptions, high-volume care, the need for rapid decisions with limited information and time, variation in staff over different shifts and fear of patient's dissatisfaction if not prescribed antibiotics. ${ }^{6}$ We propose an ED-inpatient integrated antibiotic stewardship program due to the anticipated rise in antibiotic resistance during the COVID-19 era. In addition, we call for developing locally relevant ASP guidelines, including targeted educative interventions for ED physicians on antimicrobial resistance, focusing on infection prevention and control, reserving antibiotics for critically ill Covid patients and most importantly PCT-based antibiotic review and downgrade in 48 hours.

\section{Grant Support \& Financial Disclosures: None.}

\section{REFERENCES}

1. Walley JD, Zhang Z, Wei X. Antibiotic overuse in China call for consolidated efforts to develop antibiotic stewardship programmes. Lancet Infect Dis. 2021;21(5):597. doi: 10.1016/S1473-3099(21)00196-1

2. Saleem AF, Pethani A. Antimicrobial Stewardship-Do we need it in Pakistan? J Pak Med Assoc. 2020;1:1-5.

3. Langford BJ SM, Raybardhan S, Leung V, Westwood D, MacFadden $\mathrm{DR}$, et al. Bacterial co-infection and secondary infection in patients with COVID-19: A living rapid review and meta-analysis. Clin Microbiol Infect. 2020;26(12):1622-1629. doi: 10.1016/j.cmi.2020.07.016

4. Nasir N, Rehman F, Omair SF. Risk factors for bacterial infections in patients with moderate to severe COVID-19: A case-control study. J Med Virol. 2021;93(7):4564-4569. doi: 10.1002/jmv.27000

5. Pulia M, Redwood R, May L. Antimicrobial stewardship in the emergency department. Emerg Med Clin North Am. 2018;36(4):853-872. doi: 10.1016/j.emc.2018.06.012

6. Chisholm CD CE, Nelson DR, Cordell WH. Emergency Department workplace interruptions are Emergency Physicians "Interruptdriven" \& "Multitasking"? Acad Emerg Med. 2000;7(11):1239-1243. doi:10.1111/j.1553-2712.2000.tb00469.x

Authors Contribution: MI conceived, wrote and edited the final manuscript. NNJ, SU did the literature review and manuscript writing. 